\title{
Wave packets in the anomalous Ostrovsky equation
}

\author{
E. R. Johnson $\oplus^{*}$ \\ Department of Mathematics, University College London, Gower Street, London WC1E 6BT, United Kingdom
}

(Received 23 July 2019; published 17 October 2019)

\begin{abstract}
The anomalous Ostrovsky equation, which describes waves in vertically sheared ocean flows and magnetoacoustic waves, possesses steadily propagating, finite-amplitude, localized wave-packet solutions. It is shown here that these solutions can be obtained asymptotically, using Whitham modulation theory, as the solution to a nonlinear eigenvalue problem. This allows the various wave-packet solutions to be delineated and compared to solutions of the full equations of motion. A periodic solution with an embedded wave train is also constructed.
\end{abstract}

DOI: 10.1103/PhysRevE.100.043109

\section{INTRODUCTION}

The weakly nonlinear, weakly dispersive, weakly rotating shallow water momentum equations, normalized to have maximum amplitude unity, can be written [1]

$$
\begin{aligned}
u_{t}+u u_{x}+u_{x x x} & =\gamma \epsilon v, \\
v_{x} & =\epsilon u,
\end{aligned}
$$

where $0<\epsilon \ll 1$ with $\gamma=1$ for normal dispersion and $\gamma=$ -1 for anomalous dispersion. These are usually combined to give the Ostrovsky equation [2] in the form

$$
\left(u_{t}+u u_{x}+u_{x x x}\right)_{x}=\gamma \epsilon^{2} u .
$$

This paper shows how wave-packet solutions for (2) can be constructed for anomalous dispersion using Whitham modulation theory [3,4] (WMT), removing the requirement in existing solutions [5] of obtaining boundary conditions from numerical solutions of (2). The anomalous equation arises in studies of strongly vertically sheared ocean flows [6] and of magnetoacoustic waves [7].

\section{MODULATION EQUATIONS}

The WMT equations for $(1)$ follow $[3,4]$ by introducing the long scale time and space scales $X=\epsilon x, T=\epsilon t$, considering packets consisting of waves of wavelength of order $x$, and averaging, denoted by \langle\rangle , over the carrier wave period to obtain

$$
\begin{aligned}
\langle u\rangle_{T}+(1 / 2)\left\langle u^{2}\right\rangle_{X} & =\gamma\langle v\rangle, \\
\langle v\rangle_{X} & =\langle u\rangle,
\end{aligned}
$$

which can be written as the single equation

$$
\langle u\rangle_{X T}+(1 / 2)\left\langle u^{2}\right\rangle_{X X}=\gamma\langle u\rangle .
$$

Multiplying (1a) by $u$ gives the momentum flux equation

$$
\left[(1 / 2) u^{2}\right]_{t}+\left[(1 / 3) u^{3}+u u_{x x}-(1 / 2) u_{x}^{2}-(\gamma / 2) v^{2}\right]_{x}=0 .
$$

Averaging then gives

$$
(1 / 2)\left\langle u^{2}\right\rangle_{T}+(1 / 3)\left\langle u^{3}\right\rangle_{X}-(3 / 2)\left\langle u_{x}^{2}\right\rangle_{X}=\gamma\langle u v\rangle .
$$

To leading order $\partial_{X}^{-1} u=\partial_{X}^{-1}\langle u\rangle$, and so (6) becomes

$$
(1 / 2)\left\langle u^{2}\right\rangle_{T}+(1 / 3)\left\langle u^{3}\right\rangle_{X}-(3 / 2)\left\langle u_{x}^{2}\right\rangle_{X}=\gamma\left\langle u \partial_{X}^{-1}\langle u\rangle\right\rangle .
$$

Equations (4) and (7) reproduce those obtained more formally previously [5]. The system is closed by considering the "conservation of waves" or "conservation of crests." The slowly varying density of waves or crests, the wave number $k(X, T)$ of the carrier wave, and the flux of waves, traveling with phase speed $c(X, T)$, satisfy

$$
k_{T}+(c k)_{X}=0 .
$$

In the limit $\epsilon \rightarrow 0$, Eq. (2) reduces to the Korteweg-de Vries equation with the periodic solution as that can be written in terms of the variables $r_{1} \leqslant r_{2} \leqslant r_{3}$

$$
u=u_{\min }+a \mathrm{cn}^{2}\left[2 \mathrm{~K} k(x-c t)-\theta_{0}\right],
$$

where $\mathrm{cn}$ is the Jacobi elliptic function with parameter $m=$ $\left(r_{2}-r_{1}\right) /\left(r_{3}-r_{1}\right)$ and period $2 \mathrm{~K}, k=\left(r_{3}-r_{1}\right)^{1 / 2} /[4 \sqrt{ } 3 \mathrm{~K}]$ is the local wave number, here the number of waves per unit length, $c=\left(r_{1}+r_{2}+r_{3}\right) / 6$ is the local phase speed, $u_{\min }=\left(r_{1}+r_{3}-r_{2}\right) / 2$ gives the lower envelope, $a=r_{2}-r_{1}$ is the local amplitude, and the constant $\theta_{0}$ gives a simple phase shift. The upper envelope is $u_{\max }=u_{\min }+a$ and the mean value is

$$
d=\langle u\rangle=\left(r_{1}+r_{2}-r_{3}\right) / 2+\left(r_{3}-r_{1}\right) \mathrm{E} / \mathrm{K},
$$

with $\mathrm{K}(m)$ and $\mathrm{E}(m)$ the complete elliptic integrals of the first and second kinds.

The quantities $r_{1}$ are constants for solutions of the KdV but vary slowly as functions of $X$ and $T$, as solutions of (2). Following Whitham [4], it can be shown [5] that they satisfy the WMT system

$$
\begin{aligned}
r_{i T}+Q_{i}\left(r_{1}, r_{2}, r_{3}\right) r_{i X} & =2 \gamma D, \quad \text { for } i=1,2,3, \\
D_{X} & =d,
\end{aligned}
$$


where each $r_{i}$ is a Riemann variable that propagates with characteristic velocity $Q_{i}$, given by

$$
\begin{aligned}
Q_{1} & =c-(a / 3)[\mathrm{K} /(\mathrm{K}-\mathrm{E})], \\
Q_{2} & =c-(a / 3)(1-m) \mathrm{K} /[\mathrm{E}-(1-m) \mathrm{K}], \\
Q_{3} & =c+(a / 3)(1-m) \mathrm{K} /[m \mathrm{E}] .
\end{aligned}
$$

To consider a wave packet whose envelope propagates at constant speed $s$, look for solutions of the form $r_{i}=r_{i}^{\prime}\left(X^{\prime}\right)$, $Q_{i}=Q_{i}^{\prime}\left(X^{\prime}\right)$, and $d=d^{\prime}\left(X^{\prime}\right)$, where $X^{\prime}=X-s T$. Then (11) gives, on dropping primes, the WMT for the envelope as

$$
r_{i X}=2 \gamma D /\left(Q_{i}-s\right), \quad \text { where } D_{X}=d .
$$

\section{ANOMALOUS WAVE PACKETS OF FIXED FORM}

In general the phase speed $c$ varies across the packet. However, for anomalous dispersion $(\gamma=-1)$ steadily propagating finite amplitude isolated solutions exist with $c$ constant $[5,7]$ and so attention is restricted here to this case with $s=c=$ const. Wave-packet solutions [5] consist of a rapidly varying packet of form (9) occupying a region $|X| \leqslant X_{\mathrm{e}}$ surrounded by a slowly varying outer solution in $|X|>X_{\mathrm{e}}$, that vanishes as $|X| \rightarrow \infty$. So that the inner solution matches smoothly to the outer solution, the gradient of the inner solution must vanish at the edges $X= \pm X_{\mathrm{e}}$ of the packet. So that the mass of the outer solution sums with the positive mass [5] of the inner to satisfy the "zero mass" constraint from (2),

$$
\int_{-\infty}^{\infty} u d x=0
$$

the outer solution must be negative at the edges $X= \pm X_{\mathrm{e}}$. Hence the packets terminate at $X= \pm X_{\mathrm{e}}$ in minima of the solutions of (9). For symmetric packets with a central maximum this requires the total number of wavelengths, $N$ say, in the packet to be odd. For symmetric packets with a central local minimum $N$ is even. Symmetric solutions with a central maximum are given by setting $\theta_{0}=0$ in (9) and those for a central minimum by setting $\theta_{0}=\mathrm{K}$. Admissable wave-packet solutions of (13) thus satisfy additionally

$$
\begin{array}{r}
2 \Theta_{\mathrm{e}} / \epsilon=\left[\Theta\left(X_{\mathrm{e}}\right)-\Theta\left(-X_{\mathrm{e}}\right)\right] / \epsilon=\frac{1}{\epsilon} \int_{-X_{\mathrm{e}}}^{X_{\mathrm{e}}} \Theta_{X}(X) d X \\
=\frac{1}{\epsilon} \int_{-X_{\mathrm{e}}}^{X_{\mathrm{e}}} k(X) d X=N,
\end{array}
$$

where $\Theta(X)$ is the phase within the wave packet so $\Theta_{X}(X)=$ $k(X)$ and $\Theta_{\mathrm{e}}=\Theta\left(X_{\mathrm{e}}\right)$. For a given value of $\epsilon$ only a discrete set of solutions of (13) satisfy (15) and so (13) with (15) forms a nonlinear eigenvalue problem for the wave packets. The eigensolutions are formally valid in the limit $\epsilon \rightarrow 0, N \rightarrow \infty$ with $\Theta_{\mathrm{e}}=\epsilon N / 2$ fixed but, as shown below, remain accurate even for $N=1$.

System (13) is solved most simply by integrating from $X=$ 0 to $X=X_{\mathrm{e}}$. This requires boundary conditions on the $r_{i}$ at $X=0$. For $X>X_{\mathrm{e}}$ only the variable $r_{1}=2 d$ is present [5], governed by the reduced Ostrovsky equation [8] for steady waves,

$$
\left[(d-c) d_{X}\right]_{X}+d=0,
$$

which can be integrated once to give

$$
(d-c)^{2}\left(d_{X}\right)^{2} / 2+d^{3} / 3-c d^{2} / 2=A_{0},
$$

for constant $A_{0}$. As noted above, packet solutions require $d_{\mathrm{e}}=$ $d\left(X_{\mathrm{e}}\right)<0$. For $c>0$, Eq. (17) has solutions, monotonically increasing in $X>X_{\mathrm{e}}$, asymptoting to zero to give a finite negative mass, and so forcing $A_{0}=0$ [5]. For $d_{\mathrm{e}}<c<0$ solutions of (17) are nonsingular when $d=c$ if and only if $A_{0}=-c^{3} / 6$; whence (17) becomes

$$
\frac{1}{2}\left(d_{X}\right)^{2}=-d / 3-c / 6, \quad \text { so } d_{X X}=-1 / 3,
$$

with solution

$$
d=-c / 2-\left(X-X_{0}\right)^{2} / 6, \quad X_{0}=X_{\mathrm{e}}+\sqrt{-6 d_{\mathrm{e}}-3 c} .
$$

Now consider the quantity $A$ given by

$$
A=\left(r_{1}+r_{2}-r_{3}\right)\left(r_{1}-r_{2}+r_{3}\right)\left(-r_{1}+r_{2}+r_{3}\right) / 48,
$$

which is proportional to the product of the roots of the cubic polynomial associated with (2) in the $\mathrm{KdV}(\epsilon \rightarrow 0)$ limit [4]. As $X \rightarrow X_{\mathrm{e}}^{-}$, the variables $r_{2}$ and $r_{3}$ coalesce with $r_{2}, r_{3} \rightarrow$ $2 d+a$ so $m \rightarrow 1$ and the packet terminates in a KdV solitary wave of amplitude $a$ propagating at speed $c=d+a / 3$, as expected. As also $r_{1} \rightarrow 2 d$ so $A \rightarrow d^{3} / 3-c d^{2} / 2$, and $A$ can be extended continuously into $X>X_{\mathrm{e}}$ as $A=d^{3} / 3-c d^{2} / 2$, $X \geqslant X_{\mathrm{e}}$. Integrating (2) from $X>X_{\mathrm{e}}$ to infinity gives

$$
\int_{X}^{\infty} u d X=\left.(u-c) u_{X}\right|_{X}+\epsilon^{2} u_{X X}(X) .
$$

Thus, to order $\epsilon^{2}, D=(d-c) d_{X}$ in $X \geqslant X_{\mathrm{e}}$ and (17) becomes in $X \geqslant X_{\mathrm{e}}$,

$$
\frac{1}{2} D^{2}+A=A_{0} .
$$

The modulation equations show that relation (22) in fact holds throughout the wave packet [5] also and so (22) holds for all $X$ and gives a constant of the motion. In particular, at $X=0$, where $D(0)=0$ by symmetry,

$$
A(0)=A_{0}= \begin{cases}-c^{3} / 6, & c<0 \\ 0, & c \geqslant 0\end{cases}
$$

Together with a normalization condition, chosen here to be that the wave-packet envelope has maximum value unity at $X=0$, i.e., $r_{2}+r_{3}-r_{1}=2$, Eq. (23) provides the initial conditions for a system at $X=0$ as $r_{1}=3 c-1$, and

$$
\begin{array}{ll}
c \geqslant 0: & r_{2}=3 c, \quad r_{3}=1, \\
c<0: & r_{2}, r_{3}=\frac{1}{2}(3 c+1) \mp \frac{1}{2}\left[(3 c-1)^{2}+4 c^{3}\right]^{1 / 2} .
\end{array}
$$

These conditions ensure that the wave packet joins continuously to a nonsingular outer solution. However, for $c<0$ they do not guarantee that the outer solution vanishes as $|X| \rightarrow \infty$. They do not preclude nonisolated, generalized wave-packet solutions [9] nor periodic solutions, an example of which is given in Sec. V. A sufficient condition that a wave packet does not interact with linear waves is the requirement that there exist no nondecaying linear waves with the same phase speed. Solutions of the full anomalous Ostrovsky equation propagating at constant speed $c$ satisfy

$$
\left[(u-c) u_{X}+\epsilon^{2} u_{X X X}\right]_{X}+u=0 .
$$


Linear solutions of (25) traveling with speed $c<0$, proportional to $\exp (\lambda X)$, satisfy

$$
\left(\lambda^{2}-\frac{1}{2} c\right)^{2}=\left(\frac{1}{2} c\right)^{2}-\epsilon^{2} .
$$

Constant amplitude linear waves exist if $\lambda$ is purely imaginary, i.e., $\lambda^{2}$ is real and negative, which occurs unless $\left(\frac{1}{2} c\right)^{2}>\epsilon^{2}$, i.e.,

$$
0>c>-2 \epsilon .
$$

Condition (27) does not exclude weakly nonlinear nondecaying copropagating periodic solutions [10] of (25) in $|X| \gg 1$ and so supplies only a lower bound on the wave speed for given $\epsilon$.

\section{NUMERICAL RESULTS}

The existence of two constants of the motion, $A_{0}$ and $c$, means that system (13) is effectively second order. However, it is computationally more straightforward to treat the system as fourth order and use the constants as checks on the accuracy of the integrations. In the computations reported below the constants were preserved with accuracy of order $10^{-16}$. At the edges $X= \pm X_{\mathrm{e}}$ of the wave packets the Riemann variables $r_{2}$ and $r_{3}$ coalesce with $r_{2 X}$ and $r_{3 X}$ becoming infinite. Thus for computation it is convenient to introduce an arc-length variable, $\tau$, along $r_{2}$ defined through

$$
\tau_{X}=\left[1+\left(r_{2 X}\right)^{2}\right]^{1 / 2},
$$

and solve the system

$$
\begin{aligned}
r_{i \tau} & =-2 D /\left(Q_{i}-c\right) \tau_{X}, \\
D_{\tau} & =d / \tau_{X}, \\
\Theta_{\tau} & =k(X) / \tau_{X}, \\
X_{\tau} & =1 / \tau_{X},
\end{aligned}
$$

for $\tau \geqslant 0$ subject to the initial conditions (24) and $X=0$, $\Theta=0$ at $\tau=0$.

For a given wave-packet speed $c$ this system was solved straightforwardly using the Matlab routine ode113 to determine the point, $X_{\mathrm{e}}$, of coalescence within an accuracy of $10^{-10}$. The value of $\Theta_{\mathrm{e}}=\Theta\left(X_{\mathrm{e}}\right)$ then determines $\epsilon N$ through (15). Figure 1 shows the results for $c=-0.05$, a typical integration giving here $X_{\mathrm{e}}=0.6958$ and $\Theta_{\mathrm{e}}=0.1454$. Figure 2 gives the values of $X_{\mathrm{e}}$ and $\Theta_{\mathrm{e}}$ for $-0.2 \leqslant c \leqslant 1 / 3$. The upper limit of $c \rightarrow 1 / 3^{-}$corresponds to a train in KdV solitary waves of amplitude unity on a vanishingly small shelf $d \rightarrow 0^{-}$.

Symmetric solutions of the full equation (25) for a given rotation strength $\epsilon$ can be found most simply by representing $u$ as a cosine series of period $L \gg 1$ and solving [11] for the series coefficients and speed $c$. Solutions with a central maximum ( $N$ odd) are normalized by requiring $u(0)=1$ and with a central local minimum ( $N$ even) so as to coincide at their maximum with the upper envelope of the modulation solution. The WMT packet solutions give accurate initial guesses at larger values of $c$, where the outer solution decays rapidly, and solutions for larger $\epsilon$ and smaller $c$ follow straightforwardly by continuation. Figure 3 shows wave packets for $N=7,4,2$ each with $\epsilon$ chosen as the value at

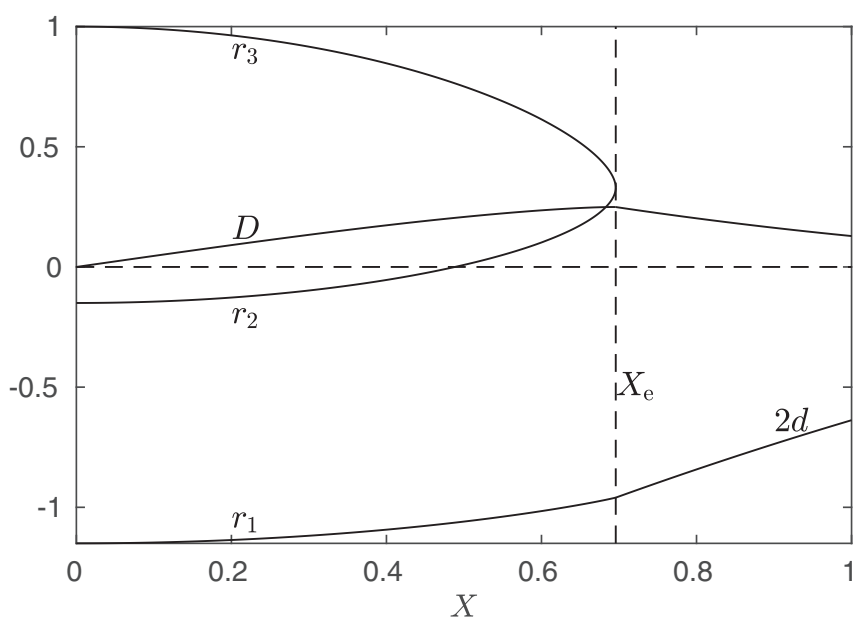

FIG. 1. Typical solution of system (29) here for $c=-0.05$ showing the variables $r_{2}$ and $r_{3}$ coalescing at $X_{\mathrm{e}}$.

the corresponding open circle in Fig. 4 to show the largest difference between the full and WMT solutions. The WMT and full solutions coincide within the interval $|X|<X_{0}$ except near $X= \pm X_{\mathrm{e}}$, where higher order terms smooth the transition between the inner and outer solutions, and the end points $X=$ $\pm X_{0}$ where long linear dispersive effects become important since each $c$ is negative. For positive speeds $c$ outer WMT and full solutions decay monotonically and coincide to graphical accuracy. Figure 4 compares the wave-packet speeds, $c$, as a function of the rotation strength, $\epsilon$, for various mode numbers, $N$, from the full numerics with those from asymptotic theories. The dash-dotted curves invert the relation $\Theta_{\mathrm{e}}(c)$ of Fig. 2 to give the speed $c$ as a function of $\epsilon$ from the WMT. The solid curves give speeds from the full numerics. All solution branches except that for $N=1$ terminate at speeds, marked in Fig. 4(a) by open circles, just greater than the lower bound given by (27), shown in Fig. 4 as a dashed line.

The distance of the termination point from the lower bound decreases with decreasing $\epsilon$ in line with the existence of weakly nonlinear nondecaying periodic waves as noted above. The mode $N=1$ is exceptional in that the solution branch exists for all $\epsilon$. The modulation solution remains remarkably accurate even though the wave packet consists of only a single peak. An alternative approximation for small $\epsilon$ can be obtained by following Hunter [12] and considering a single $\mathrm{KdV}$ soliton on a weak, variable background. The zero mass
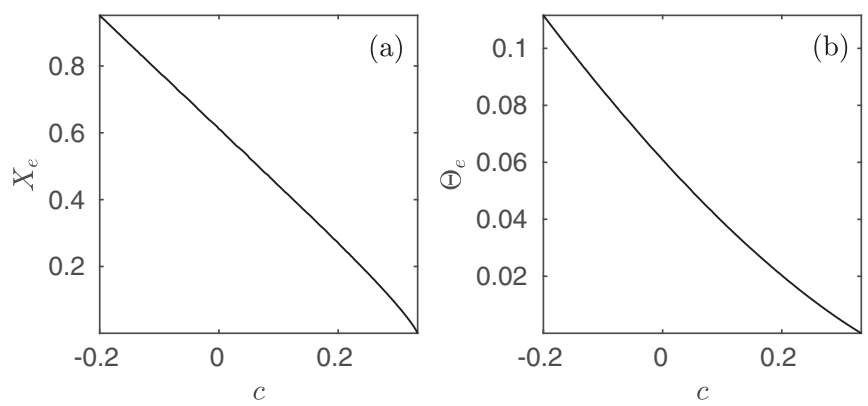

FIG. 2. Values of (a) the wave-packet halfwidth $X_{\mathrm{e}}$ and (b) the edge phase $\Theta_{\mathrm{e}}$, for speeds $-0.2 \leqslant c \leqslant 1 / 3$. 

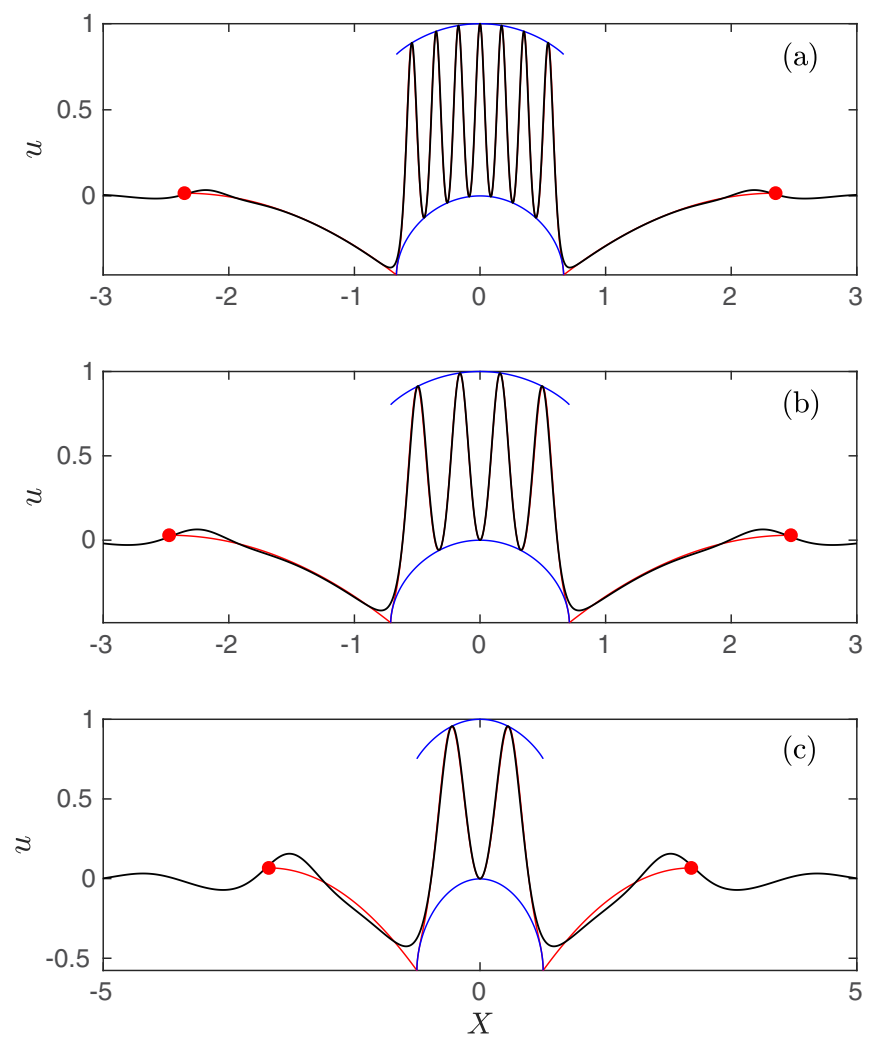

FIG. 3. Wave packets with (a) $N=7$ for $\epsilon=0.01954$, (b) $N=4$ for $\epsilon=0.03742$, and (c) $N=2$ for $\epsilon=0.09402$. The thin curve (red online) gives the WMT solution [with speeds (a) $c=-0.032$, (b) $c=-0.059$, and (c) $c=-0.134]$. The thicker curves give the upper and lower envelopes (blue online), and the full solution [with speeds (a) $c=-0.034$, (b) $c=-0.066$, and (c) $c=-0.166]$. The outer WMT solutions are terminated at $X= \pm X_{0}$, with (a) $X_{0}=$ \pm 2.3534 , (b) $X_{0}= \pm 2.4753$, and (c) $X_{0}= \pm 2.8031$ marked by solid dots.

constraint then gives, in the present notation, $c=1 / 3-4 \epsilon$, shown dotted in Fig. 4.

The large $\epsilon$ limit of (2) corresponds to weakly nonlinear flow [1] and so the solutions can be described in terms of the nonlinear Schrödinger (NLS) equation. A solution of (2) accurate for $\epsilon \gg 1$ to order $\epsilon^{-2}$ can be written [1] as

$$
\begin{aligned}
u= & 2 a_{0} \operatorname{sech}\left[K_{0}\left(X-c_{\mathrm{g}} T\right)\right] \cos \left[k_{0}\left(X-c_{\mathrm{c}}\right) T\right] \\
& +2 a_{0}^{2} \chi \operatorname{sech}^{2}\left[K_{0}\left(X-c_{\mathrm{g}} T\right)\right] \cos \left[2 k_{0}\left(X-c_{\mathrm{c}}\right) T\right],
\end{aligned}
$$

where $\chi=2 k_{0}^{2} /\left(12 k_{0}^{4}+3 \gamma\right), c_{\mathrm{g}}$ is the envelope speed, and $c_{\mathrm{c}}$ is the carrier wave phase speed. For the packets of fixed form here, $c_{\mathrm{g}}$ and $c_{\mathrm{c}}$ coincide and, again to order $\epsilon^{-2}$, it can be shown [13] that

$$
\begin{aligned}
k_{0}=\epsilon^{-1 / 2}\left(1-a_{0}^{2} / 72 \epsilon^{2}\right), \quad K_{0} & =a_{0} / 6 \epsilon^{3 / 2}, \\
c & =c_{\mathrm{g}}=c_{\mathrm{c}}=-2 \epsilon+a_{0}^{2} / 9 \epsilon,
\end{aligned}
$$

where the normalization $u(0,0)=1$ here determines $a_{0}$ from

$$
2 a_{0}+2 a_{0}^{2} \chi=1 \text {, i.e., } a_{0}=(\sqrt{1+2 \chi}-1) / 2 \chi .
$$

The speed prediction from (31) and (32) is shown as a thickened solid curve (red online) for $\epsilon>0.5$ in Fig. 4(b) and
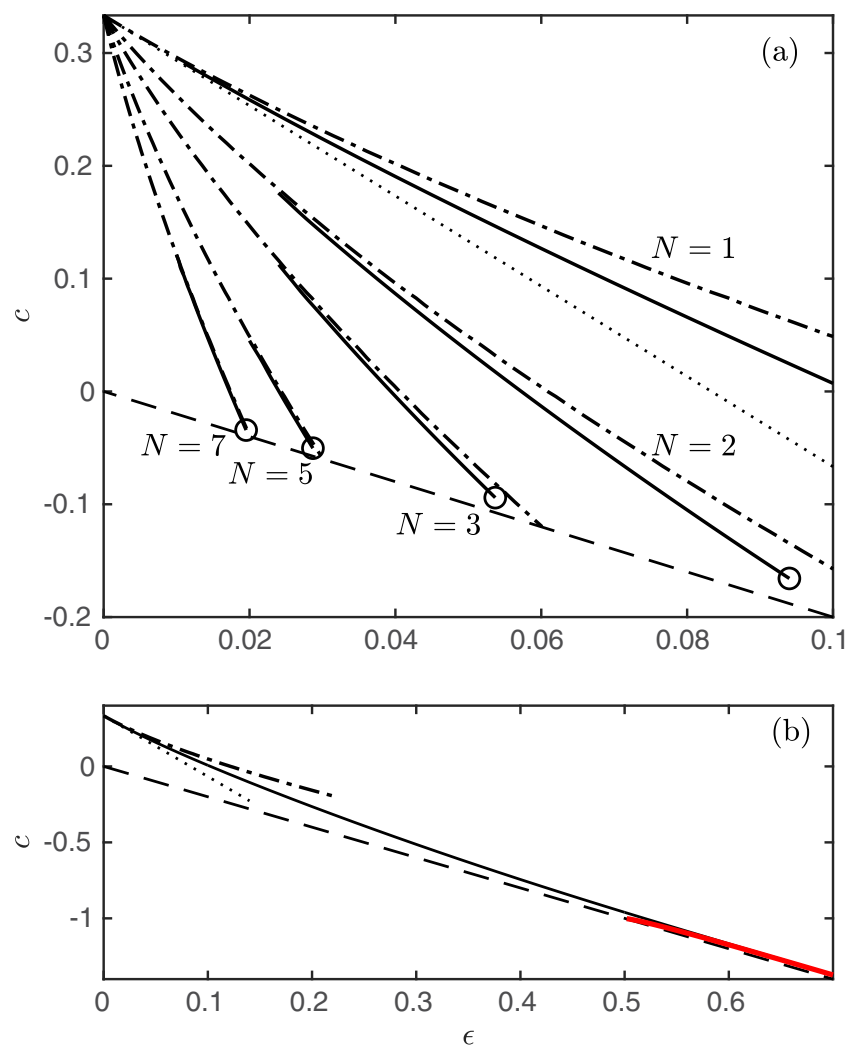

FIG. 4. Wave-packet speeds, $c$, as a function of the rotation strength, $\epsilon$, for various mode numbers, $N$. The dashed line gives the lower bound from (27). The dash-dotted curves give the speeds derived from Fig. 2 and (15) and the solid curves give the full solutions of (25). The dotted curve gives the single peak speed for $N=1$. The thicker solid curve in (b) (red online) gives the speed for large $\epsilon$ from the NLS approximation.

remains accurate with decreasing $\epsilon$ until near $\epsilon=0.5$, where $\chi$ is singular due to resonance between the first and second order terms in (30) [13]. Figure 5 shows a wave packet for $\epsilon=0.8$ from the continuation of the $N=1$ solution. Even at this value of $\epsilon^{-1}=1.25$, the second order NLS solution (30) is remarkably accurate, capturing the up-down asymmetry of the full solution.

\section{DISCUSSION}

The transformation $\hat{u}=-u, \hat{X}=-X$ maps Eq. (2) in the limit $\epsilon \rightarrow 0$, in terms of $X$ and $T$ into the anomalous $(\gamma=-1)$ reduced Ostrovsky equation with normal dispersion $(\gamma=1)$,

$$
\left(\hat{u}_{T}+\hat{u} \hat{u}_{\hat{X}}\right)_{\hat{X}}=\hat{u} .
$$

Differentiating (33) once and rearranging shows that $F=$ $\left(1+3 u_{X X}\right)^{1 / 3}$ is a conserved density in that

$$
F_{T}+(u F)_{X}=0,
$$

and it follows [8] that solutions of the reduced anomalous Ostrovsky equation break if at any time $u_{X X}<-1 / 3$ and do not break for initial conditions where $u_{X X}>-1 / 3$ everywhere. Equation (18) shows that the outer solution for $c<0$ lies exactly on $F=0$ and so it appears that the generation of a 


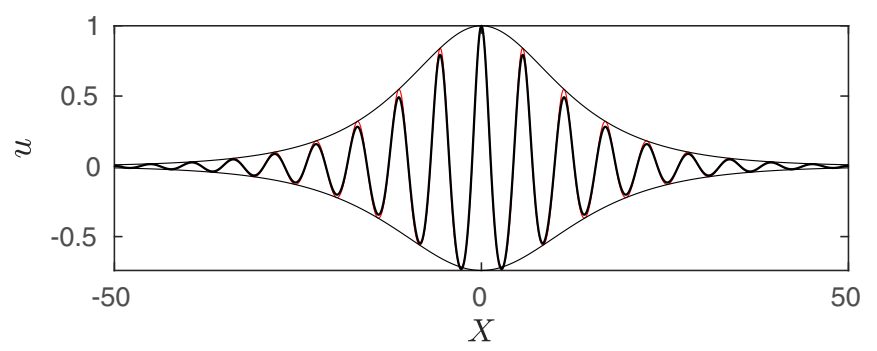

FIG. 5. Wave packet from the continuation of the $N=1$ solution. Here $\epsilon=0.8$ and $c=-1.57306$. The NLS approximation is given by the thin curve (red online) and the upper and lower NLS envelopes are also shown. The thicker curve gives the full solution.

wave packet in the evolution of solutions of the full equations (2) is associated with breaking of long wave solutions.

The wave-packet solutions here persist when set as initial conditions for numerical integrations of (2) and so are likely to be stable solutions of the equations of motion. However, for small $\epsilon$ the numerical evolution of a single hump initial condition consists of the usual $\mathrm{KdV}$ evolution into solitary waves and a dispersing tail and then the subsequent evolution into $N=1$ anomalous Ostrovsky solitons.

When $c<0$, Eq. (19) gives $d_{X}=0$ at $X=X_{0}$ and so, by (21) to order $\epsilon^{2}, D\left(X_{0}\right)=0$; i.e., the mass of the wave packet

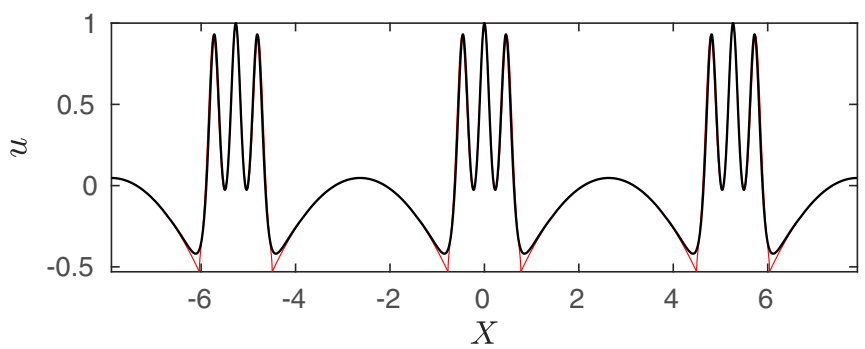

FIG. 6. Periodic wave train containing $N=3$ wave packets. Here $\epsilon=0.05369, c=-0.09414$ and the period is $2 X_{0}=5.2664$. The thin curve (red online) is the WMT solution and the thicker curve is the full solution.

over the interval $|X| \leqslant X_{0}$ vanishes. The wave-packet solution can thus be extended as periodic with period $2 X_{0}$ and mass vanishing over each period, as required by (2) integrated over one period. Figure 6 gives the periodic wave train formed by so extending the $N=3$ packet corresponding to the open circle in Fig. 4(a). Away from the packet the WMT and full solutions coincide almost exactly as the outer solution (19) satisfies the full equations identically. Since the wave train is no longer constrained to vanish at large $X$ the constraint (27) no longer applies and wave train solutions exist for $c<-2 \epsilon$.
[1] A. J. Whitfield and E. R. Johnson, Rotation-induced nonlinear wavepackets in internal waves, Phys. Fluids 26, 056606 (2014).

[2] L. A. Ostrovsky, Nonlinear internal waves in a rotating ocean, Oceanology 18, 199 (1978).

[3] G. B. Whitham, Non-linear dispersive waves, Proc. R. Soc. London A 283, 238 (1965).

[4] G. B. Whitham, Linear and Nonlinear Waves (Wiley, New York, 1974).

[5] A. J. Whitfield and E. R. Johnson, Whitham modulation theory for the Ostrovsky equation, Proc. R. Soc. A 473, 20160709 (2017).

[6] A. Alias, R. H. J. Grimshaw, and K. R. Khusnutdinova, Coupled Ostrovsky equations for internal waves in a shear flow, Phys. Fluids 26, 126603 (2014).

[7] M. A. Obregon and Y. A. Stepanyants, Oblique magnetoacoustic solitons in a rotating plasma, Phys. Lett. A 249, 315 (1998).
[8] R. H. J. Grimshaw, K. Helfrich, and E. R. Johnson, The reduced Ostrovsky equation: Integrability and breaking, Stud. Appl. Math. 129, 414 (2012).

[9] J. P. Boyd, in Weakly Nonlocal Solitary Waves and BeyondAll-Orders Asymptotics, edited by M. Hazewinkel (Kluwer Academic Publishers, Dordrecht, 1998).

[10] J. P. Boyd and G. Y. Chen, Five regimes of the quasi-cnoidal, steadily translating waves of the rotation-modified Korteweg-de Vries ("Ostrovsky") equation, Wave Motion 35, 141 (2002).

[11] E. R. Johnson and D. E. Pelinovsky, Orbital stability of periodic waves in the class of reduced Ostrovsky equations, J. Diff. Eq. 261, 3268 (2016).

[12] J. K. Hunter, in Computational Solution of Nonlinear Systems of Equations, edited by E. Allgower, Lectures in Applied Mathematics Vol. 26 (American Mathematical Society, 1990), p. 762.

[13] R. Grimshaw, Y. Stepanyants, and A. Alias, Formation of wave packets in the Ostrovsky equation for both normal and anomalous dispersion, Proc. R. Soc. A 472, 20150416 (2016). 